ANNALES

POLONICI MATHEMATICI

XVIII (1966)

\title{
The inverse Meijer transform of the $G$-function
}

\author{
by S. M. MathuR (Jodhpur)
}

1. Introduction. In the present paper we consider the integral transform

$$
F(p)=p \int_{0}^{+\infty}(p t)^{-k-\frac{1}{2}} e^{-\frac{1}{2} p t} W_{k+\frac{1}{2}, m}(p t) f(t) d t,
$$

of a function $F \in L(0, \infty)$, where $\operatorname{Re} p>0$ and $W$ denotes Whittaker's function. Transform (1) was defined first by Meijer in [4] (formula (5), p. 730); if $k= \pm m$, it reduces to the classical Laplace transform.

The purpose of this paper is to determine the original function whose transform (1) is $G\left(a p^{n / s}\right)$, where $n$ and $s$ are natural, and $G$ denotes Meijer's function (for the definition, see e.g. [2], p. 207). Next, we obtain as special cases some earlier results of Ragab ([6], [7]) and Saxena ([8]). Our method is based on the formula

$$
\begin{array}{r}
\int_{0}^{+\infty} t^{l-1} e^{-\frac{1}{2} p t} W_{k+\frac{1}{2}, m}(p t) d t=p^{-l} \Gamma\left(l+m+\frac{1}{2}\right) \Gamma\left(l-m+\frac{1}{2}\right) / \Gamma\left(l-l_{t}+\frac{1}{2}\right) \\
\left(\operatorname{Re} p>0, \operatorname{Re}\left(l \pm m+\frac{1}{2}\right)>0\right)
\end{array}
$$

(see e.g. [3], formula (16), p. 216) and on the multiplication formula of Gauss and Legendre for the Gamma function (see e.g. [2], formula (11), p. 4).

My thanks are due to Dr. R. K. Saxena for his help and interest in the preparation of this paper.

2. The result. Let us prove the following

Theorem. Let $\alpha, \beta, \gamma, \delta, n, s$ be integers such that

$$
0 \leqslant \alpha \leqslant \delta, \quad 0 \leqslant \beta \leqslant \gamma, \quad n>0, \quad s>0, \quad \alpha+\beta>\frac{1}{2} \gamma+\frac{1}{2} \delta+\frac{1}{2} n / 8 .
$$

Further let $a_{1}, \ldots, a_{y}, b_{1}, \ldots, b_{\delta}, a, p, \sigma, k, m$ be arbitrary parameters satisfying the relations

$$
\begin{aligned}
& a_{f}-b_{h} \neq v \quad(j=1, \ldots, \beta ; \hbar=1, \ldots, \alpha ; \nu=1,2, \ldots), \\
& n a_{j}-s(\sigma-2 k) \neq v s n-x n+\lambda s \\
& \quad(j=1, \ldots, \beta ; x=0, \ldots, s-1 ; \lambda=0, \ldots, n-1 ; \nu=1,2, \ldots),
\end{aligned}
$$




$$
\operatorname{Re} p>0, \quad \operatorname{Re}\left(\sigma-k \pm m-\frac{n}{s} a_{j}+\frac{n}{s}\right)>0 \quad(j=1, \ldots, \beta)
$$

and either

$$
|\arg a|<\left(\alpha+\beta-\frac{1}{2} \gamma-\frac{1}{2} \delta-\frac{1}{2} n / s\right) \pi, \quad a+\beta<\frac{1}{2} \gamma+\frac{1}{2} \delta+\frac{1}{2} n / s
$$

or

where

$$
\begin{aligned}
& \operatorname{Re}\left[\left(k-\sigma \pm m+\frac{1}{2} n-1\right)(s \delta-s \gamma-n)+\right. \\
& \left.\quad+n\left(s+\frac{1}{2} \gamma-\frac{1}{2} \delta-n \delta-s A-n-{ }_{2}^{3}\right)\right]>0,
\end{aligned} \quad \begin{aligned}
& |\arg a|=\left(\alpha+\beta-\frac{1}{2} \gamma-\frac{1}{2} \delta-\frac{1}{2} n / s\right) \pi, \quad a+\beta=\frac{1}{2} \gamma+\frac{1}{2} \delta+\frac{1}{2} n / s,
\end{aligned}
$$

$$
A=\sum_{j=1}^{\gamma} a_{j}-\sum_{j=1}^{\delta} b_{j}-\frac{1}{2} \gamma+\frac{1}{2} \delta-1 .
$$

If $\Delta(n ; a)$ denotes the sequence of the parameters $a / n,(a+1) / n, \ldots,(a+n-1) / n$ then

$$
\int_{0}^{+\infty} t^{\sigma-k-\frac{\mathrm{s}}{2}} e^{-\frac{1}{2} p t} W_{k+\frac{1}{2}, m}(p t) \times
$$

$$
\begin{gathered}
\times G_{s \gamma+2 n, s \delta+n}^{s a+n, s \beta}\left(\frac{n^{n} a^{s}}{s^{s(\delta-\gamma)} t^{n}} \mid \begin{array}{c}
\Delta\left(s ; a_{1}\right), \ldots, \Delta\left(s ; a_{\gamma}\right), \Delta(n ; \sigma-k+m), \Delta(n ; \sigma-k-m) \\
\Delta(n ; \sigma-2 k), \Delta\left(s ; b_{1}\right), \ldots, \Delta\left(s ; b_{\delta}\right)
\end{array}\right) d t \\
=(2 \pi)^{\frac{1}{2}-\frac{1}{2} n+(s-1)\left(a+\beta-\frac{1}{2} \gamma-\frac{1}{2} \delta\right)} n^{\sigma-\frac{1}{2}} s^{A} p^{k-\sigma+\frac{1}{2}} G_{\gamma, \delta}^{a, \beta}\left(a p^{n / s} \mid \begin{array}{l}
a_{1}, \ldots, a_{\gamma} \\
b_{1}, \ldots, b_{\delta}
\end{array}\right) .
\end{gathered}
$$

Proof. Let us use the definition of the $G$-function as a contour integral (see e.g. [2], p. 207), where the path $L$ of integration runs from $-i \infty$ to $+i \infty$ so that all the poles of $\Gamma\left(b_{j}-\zeta\right)$ for $j=1, \ldots, \alpha$ are to the right and all the poles of $\Gamma\left(1-a_{h}+\zeta\right)$ for $h=1, \ldots, \beta$ are to the left of $L$. Then the $G$-functions in (8) are defined provided the conditions (3), (4), (5) and (7) are fulfilled. In view of the asymptotic behaviour of the functions $W$ and $G$ in the neighbourhood of the points 0 and $\infty$ (see [2], §§ 6.9,6.13.1, 5.4.1 and [5], § 18), the integral on the left-hand side of (8) is convergent if we assume (6).

Now, we may substitute the value of the $G$-function on the left-hand side of (8) in terms of the contour integral mentioned above. Then we interchange the order of integration applying a well-known theorem concerning the inversion of a repeated infinite integral (see e.g. [1], p. 504). Let us use in turn formula (2) with $l=\sigma-k-n \zeta-\frac{1}{2}$, which is permissible, because we may assume that $\operatorname{Re} \zeta \leqslant \varepsilon+(1 / s)\left(\operatorname{Re} a_{f}-1\right)$, where $\varepsilon>0$ is sufficiently small. Now, applying the multiplication formula of Gauss and Legendre to the Gamma function, mentioned above, we obtain a contour integral which is equal to the right-hand side of (8), as is easily seen.

Similarly we can obtain formula (8) under conditions (7') instead of (7), and then our Theorem is established. 
3. Particular cases. (i) Let $a=\delta=2, \beta=\gamma=0, \quad b_{1}=\frac{1}{2} \nu$, $b_{2}=-\frac{1}{2} \nu$. If $n$ and $s$ are natural, $2 s>n,|\arg a|<\frac{1}{2}\left(1-\frac{1}{2} n / s\right) \pi, \operatorname{Re} p>0$, and $F(p)=2 p^{1-\sigma} K_{\nu}\left(a p^{n / 2 s}\right)$, then using (4) from [2], p. 216, one obtains

$$
\begin{aligned}
f(t)=(2 \pi)^{\frac{1}{2}+\frac{1}{2} n-s-s} n^{\frac{1}{2}-\sigma} t^{\sigma-1} \times \\
\times G_{2 n, 2 s+n}^{2 s+n, 0}\left(\frac{n^{n} a^{2 s}}{(2 s)^{2 s} t^{n}} \mid \begin{array}{l}
\Delta(n ; \sigma-k+m), \Delta(n ; \sigma-k-m) \\
\Delta\left(s ; \frac{1}{2} \nu\right), \Delta\left(s ;-\frac{1}{2} \nu\right), \Delta(n ; \sigma-2 k)
\end{array}\right),
\end{aligned}
$$

and, in particular, for $n=1, \sigma=1-\frac{1}{2} \alpha-\frac{1}{2} \beta, k=m$, after replacing $v$ by $s(\beta-\alpha)$ and applying (14) from [2], p. 210, Ragab's formula published in [6] (formula (17), p. 395).

(ii) Let $\alpha=\delta=4, \quad \beta=0, \quad \gamma=2, \quad a_{1}=1+\lambda, \quad a_{2}=1-\lambda, \quad b_{1}=\frac{1}{2}$, $b_{2}=1, \quad b_{\mathrm{a}}=\frac{1}{2}+\mu, b_{4}=\frac{1}{2}-\mu$. If $n$ and $s$ are natural, $2 s>n,|\arg a|$ $<\frac{1}{2}\left(1-\frac{1}{2} n / s\right) \pi, \operatorname{Re} p>0$, and $F(p)=p^{1-\sigma} W_{\lambda, \mu}\left(a p^{\frac{1}{2} n / s}\right) W_{-\lambda, \mu}\left(a p^{\frac{1}{2} n / s}\right)$, then using (38) from [2], p. 218, one obtains

$$
\begin{aligned}
& f(t)=\pi^{-\frac{1}{2}}(2 \pi)^{\frac{1}{2}+\frac{1}{2} n-s} n^{\frac{1}{2}-\sigma} s^{\frac{1}{2}} t^{\sigma-1} \times \\
& \times G_{2 s+2 n, 4 s+n}^{4 s+n, 0}\left(\frac{n^{n} a^{2 s}}{(2 s)^{2 s} t^{n}} \mid \begin{array}{l}
\Delta(s ; 1 \pm \lambda), \Delta(n ; \sigma-k+m), \Delta(n ; \sigma-k-m) \\
\Delta\left(s ; \frac{1}{2}\right), \Delta\langle s ; 1), \Delta\left(s ; \frac{1}{2} \pm \mu\right), \Delta(n ; \sigma-2 k)
\end{array}\right),
\end{aligned}
$$

and, in particular, for $n=2, \sigma=1, k=m, \lambda=n l, \mu=n v$, after applying (14) from [2], p. 210, Ragab's formula published in [7] (formula (14), p. 581 ).

(iii) Similarly, as in the previous case, one proves that if $n$ and $s$ are natural, $2 s>n$, the parameters $a_{1}=1+\lambda, b_{h}(h=1,2,3)$, equal to $\frac{1}{2}, 1, \frac{1}{2}+\mu$ respectively, satisfy (4) and (5), $|\arg a|<\frac{1}{2}\left(1-\frac{1}{2} n / s\right) \pi$, $\operatorname{Re} p>0, \operatorname{Re}(\sigma-k \pm m-n \lambda / s)>0$ and $F^{\prime}(p)=p^{1-\sigma} W_{\lambda_{\mu, \mu}}\left(a p^{\frac{1}{2} n / s}\right) \lambda I_{-\lambda, \mu}\left(a p^{\frac{1}{2} n / s}\right)$, then

$$
\begin{gathered}
f(t)=\pi^{-\frac{1}{2}}(2 \pi)^{\frac{1}{2}+\frac{1}{2} n-s} n^{\frac{1}{2}-\sigma} s^{\frac{1}{2}}\left\{\Gamma(1+2 \mu) / \Gamma\left(\frac{1}{2}+\mu-\lambda\right)\right\} t^{\sigma-1} \times \\
\times G_{2 s+2 n, 4 s+n}^{s s+n, s}\left(\frac{n^{n} a^{2 s}}{(2 s)^{2 s} t^{n}} \mid \begin{array}{l}
\Delta(s ; 1 \pm \lambda), \Delta(n ; \sigma-k+m), \Delta(n ; \sigma-k-m) \\
\Delta(n ; \sigma-2 k), \Delta\left(s ; \frac{1}{2}\right), \Delta(s ; 1), \Delta\left(s ; \frac{1}{2} \pm \mu\right)
\end{array}\right) .
\end{gathered}
$$

(iv) If $n$ and $s$ are natural, $4 s>n$, the parameters $a_{1}=1+\lambda, b_{n}$ $(h=1,2,3,4)$, equal to $\frac{1}{2}, 1, \frac{1}{2} \pm \mu$ respectively, satisfy (4) and (5), $|\arg a|<\frac{1}{2}\left(2-\frac{1}{2} n / s\right) \pi, \quad \operatorname{Re} p>0, \operatorname{Re}(\sigma-k \pm m-n \lambda / s)>0$ and $F(p)$ $=p^{1-\sigma} W_{\lambda, \mu}\left(2 i a p^{n / s}\right) W_{\lambda, \mu}\left(-2 i a p^{n / s}\right)$, then

$$
\begin{aligned}
& f(t)=\pi^{-\frac{1}{2}}(2 \pi)^{\frac{3}{2}+\frac{1}{2} n-s} s^{\frac{1}{2}}\left\{1 / \Gamma\left(\frac{1}{2}+\mu-\lambda\right) \Gamma\left(\frac{1}{2}-\mu-\lambda\right)\right\} t^{\sigma-1} \times \\
& \times G_{2 s+2 n, 48+n}^{4 s+n, s}\left(\frac{n^{n} a^{2 s}}{s^{2 s} t^{n}} \mid \begin{array}{l}
\Delta(s ; 1 \pm \lambda), \Delta(n ; \sigma-k+m), \Delta(n ; \sigma-k-m) \\
\Delta\left(s ; \frac{1}{2}\right), \Delta(s ; 1), \Delta\left(s ; \frac{1}{2} \pm \mu\right), \Delta(n ; \sigma-2 k)
\end{array}\right) .
\end{aligned}
$$

(v) If $k=m$ then (8) reduces to a result given by Saxena in [8] (formula (11), p. 40). 


\section{Relerences}

[1] T. J. I'a Bromwich, An introduotion to the theory of infinite series, II ed., London, 1950.

[2] A. Erdélyi et al., Higher transcendental functions $I$, New York, 1953.

[3] - Tables of integral transforms I, Now York, 1954.

[4] C. S. Meijer, Eine neue Erweiterung der Laplace-Transformation I, Proc. Kon. Ned. Akad, v. Wetensch. 44 (1941), pp. 727-737.

[6] - On the G-function VI-VIII, Proc. Kon. Ned. Akad. v. Wetensch. 49 (1946), pp. 936-943, 1063-1072 and 1165-1175.

[6] F. M. Ragab, The inverse Laplace transform of the modified Bessel function $K_{m n}\left(a^{1 / 2 m} p^{1 / 2 m}\right)$, Journ. London Math. Soc. 37 (1962), pp. $391-402$.

[7] - The inverse Laplace transform of the product of two Whittaleer functions, Proc. Cambr. Phil. Soc. 58 (1962), pp. 580-682.

[8] R. K. Saxena, Some theorems in operational oalculus and infinite integrals involving Bessel functions and G-functions, Proc. Nat. Inst. Sci. India 27A (1961), pp. 38-61.

DEPARTMENT OF MATHEMATICS, JODHPUR UNIVERSITY

Reģu par la Rédaction le 2.12. 1964 